

\title{
OPTIMIZACIÓN DE LA EFICIENCIA ENERGÉTICA APLICANDO CONFORTTÉRMICO ADAPTATIVO ENUN EDIFICIO DE OFICINAS PÚBLICO EN SAN JUAN-ARGENTINA
}

\section{ENERGY EFFICIENCY OPTIMIZATION THROUGH ADAPTIVE THERMAL COMFORT IN A PUBLIC OFFICE BUILDING IN SAN JUAN, ARGENTINA}

\author{
Bruno Damián Arballo \\ Arquitecto Becario CONICET \\ Estudiante de Doctorado del Instituto Regional de Planeamiento \\ y Hábitat, Universidad Nacional de San Juan, \\ Consejo Nacional de Investigaciones Científicas y Técnicas \\ San Juan Argentina \\ https://orcid.org/0000-0002-6121-549X \\ arballobruno@gmail.com
}

Ernesto Kuchen

Doctor

Investigador y docente, Instituto Regional de Planeamiento y Hábitat, Universidad Nacional de San Juan, Consejo Nacional de Investigaciones Científicas y Técnicas

$$
\text { San Juan, Argentina }
$$

http://orcid.org/0000-0001-6649-6932

ernestokuchen@faud.unsj.edu.ar

\author{
Daniel Chuk \\ Doctor \\ Docente del Instituto de Investigaciones Mineras, Universidad \\ Nacional de San Juan \\ San Juan, Argentina \\ https://orcid.org/0000-0003-3171-9571 \\ dchuk@unsj.edu.ar
}

\section{RESUMEN}

Las edificaciones son responsables del $40 \%$ de la demanda mundial de energía y de emisiones de CO2. En Argentina, los edificios representan más del $40 \%$ del consumo total anual de energía. Las estrategias de ahorro de energía pueden provocar disconformidad térmica en los habitantes de los edificios. El objetivo de este trabajo es optimizar las temperaturas de "set point" diarias basadas en los perfiles de ocupación y la evolución del clima exterior para encontrar el equilibrio entre la eficiencia energética y el confort térmico de un edificio de oficinas público en San Juan, Argentina. El modelo tiene en cuenta la capacidad de adaptación térmica de los habitantes considerando la variación de la temperatura exterior. El método propuesto combina el análisis de monitoreo in situ,

que introduce datos subjetivos de ocupación, con la herramienta de simulación energética para edificios Energy Plus. Los resultados de la optimización muestran alrededor de un $26 \%$ de ahorro de energía, manteniendo condiciones aceptables de confort térmico.

Palabras clave

eficiencia energética, confort térmico, edificio de oficinas, optimización de edificios, adaptación térmica.

\section{ABSTRACT}

Buildings are responsible for $40 \%$ of global energy demand and $\mathrm{CO} 2$ emissions. In Argentina, buildings represent more than $40 \%$ of total annual energy consumption. Energy-saving strategies can cause thermal discomfort for building inhabitants. The aim of this research is to optimize daily set point temperatures based on occupancy profiles and changes in outdoor weather to find a balance between energy efficiency and thermal comfort in a public office building in San Juan, Argentina. The model takes into account the thermal adaptation capacity of the inhabitants considering outdoor temperature variation. The proposed method combines spot monitoring analysis, which introduces subjective occupancy data, with the Energy Plus building energy simulation tool. Optimization results show around $26 \%$ energy savings, while maintaining acceptable thermal comfort conditions. 


\section{INTRODUCCIÓN}

A nivel mundial las edificaciones representan alrededor del $40 \%$ del uso de energía, constituyéndose naturalmente en potenciales escenarios de ahorro de energía y emisiones (Yang y Wang, 2013; Pérez-Lombard, Ortiz y Pout, 2008; Moroşan et al., 2010). El ser humano moderno pasa la mayor parte de su tiempo en ambientes interiores (Van Hoof, Mazej y Hensen, 2010), de un 80\% a un 90\% (Environmental Protection Agency, 2014). Múltiples investigaciones han validado al confort térmico como una de las variables que mayormente afectan al confort en espacios interiores y a la eficiencia energética de los edificios (Holmes y Hacker, 2007; Pablo-Romero, Pozo-Barajas y Yñíguez, 2017; Derbez et al., 2014; Tweed et al., 2013; Al-Ajmi, 2010; Nguyen, Reiter y Rigo, 2014). Concretamente, según encuestas realizadas en un estudio a más de 1000 empleados de empresas, en Argentina el $41 \%$ del tiempo que las personas están despiertas en el día lo destinan a trabajos de oficina (M.I.S Staples, 2012). Así también, diversos estudios internacionales validan la perspectiva de confort térmico adaptativo como estrategia de ahorro de energía fundamental en edificios de oficina (Dounis y Caraiscos, 2009; Chandel, Sharma y Marwah, 2016; Sánchez-García et al., 2017), en la medida en que conduce a ahorros de entre un 30 a un $60 \%$, en especial cuando se tiene en cuenta la evolución del clima exterior.

A nivel local, trabajos anteriores desarrollados en el proyecto PICT2009-0014 Res.N³04/2010, "EEC, Eficiencia Energética y Confort en Espacios de Trabajo", en la ciudad de San Juan Argentina, fundamentan la disconformidad térmica de los habitantes frente a su ambiente laboral. Los perfiles del voto de confort (CV) de los habitantes, tal como se muestra en la Figura 1, indican una sensación térmica de frío $(-0,18)$ para verano, sobre la escala de 7 puntos de ASHRAE (Alonso Frank et al., 2015). La línea de tendencia con pendiente $b$ (capacidad de adaptación de los habitantes) encontrada para $C V$ en función de la temperatura de neutralidad $\left(t_{n}\right)$ es inversamente proporcional a la esperada (Figura 1). La temperatura deseada por los habitantes se encuentra por encima de la temperatura constante que entrega el equipo de climatización (temperatura interior de set point en verano), lo cual provoca que el $44 \%$ del consumo de energía del edificio estudiado se emplee en restituir la situación de confort de sus habitantes.

Es de especial relevancia considerar la adaptabilidad de los habitantes y las variables climáticas del sitio en tiempo real, especialmente $t_{e}$ (temperatura exterior), a fin de delimitar rangos de aceptación y variación de la temperatura interior de set point para el correcto funcionamiento de los equipos de climatización. Los sistemas de climatización total requieren de verificación en función a los estándares internacionales para definir su potencia y parámetros de control, pero también de

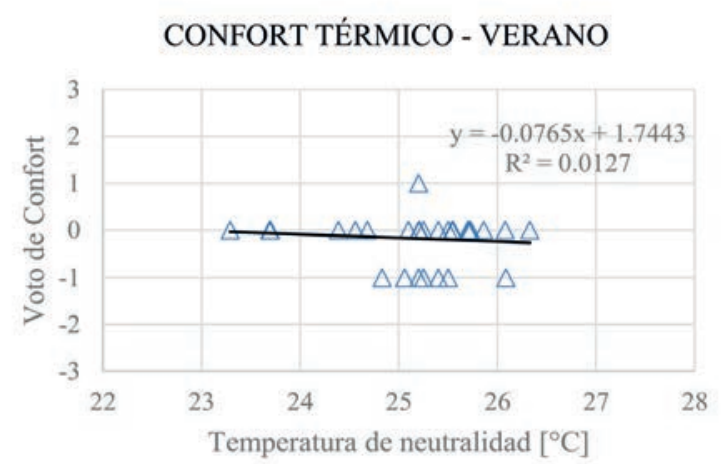

Figura 1. Confort térmico de un día típico de verano para el estudio de caso basado en el análisis de ISSO 7730. Fuente: Elaborado en base a datos publicados en Alonso Frank et al., 2015.

la actualización y validación en base a las exigencias del sitio y a la capacidad de adaptación potencial de los habitantes.

En este sentido, es necesaria una optimización del funcionamiento del edificio (EnBop, 2008) y el desarrollo de métodos de optimización en tiempo real entre eficiencia energética (ahorro energético) y confort térmico de los habitantes.

Estas variables se encuentran en conflicto, dado que un ahorro significativo de energía en el sistema energético de climatización puede resultar en condiciones internas de disconformidad térmica para los habitantes. A su vez, el consumo energético de los edificios depende significativamente de las demandas del ambiente interior, el cual afecta a la salud, rendimiento y confort (Bliuc, Rotberg y Dumitrescu, 2007). El objetivo del presente trabajo es optimizar el set point diario de un día típico de verano para una oficina tipo del edificio en estudio, en base a los perfiles de los habitantes y en función de la variación de la temperatura exterior.

A partir del estudio de cada una de estas variables y de datos reales tomados mediante mediciones de campo en los edificios (Kuchen, 2008; Kuchen, Plesser y Fisch, 2012) se definen las características del problema de optimización. Las variables de entrada o funcionales matemáticos necesarios para la optimización se construyen a partir de los modelos de confort térmico y de energía. La aplicación del modelo de optimización adaptativo-variable lleva a ahorros significativos de energía, en equilibrio con un apropiado confort térmico de los habitantes.

Este trabajo de investigación se enmarca dentro de un proyecto de desarrollo social y tecnológico (Proyecto EECOM, 2016) en curso, titulado "Eficiencia Energética y Confort en Edificios Públicos mediante Optimización Multiobjetivo", en la ciudad de San Juan, Argentina. 

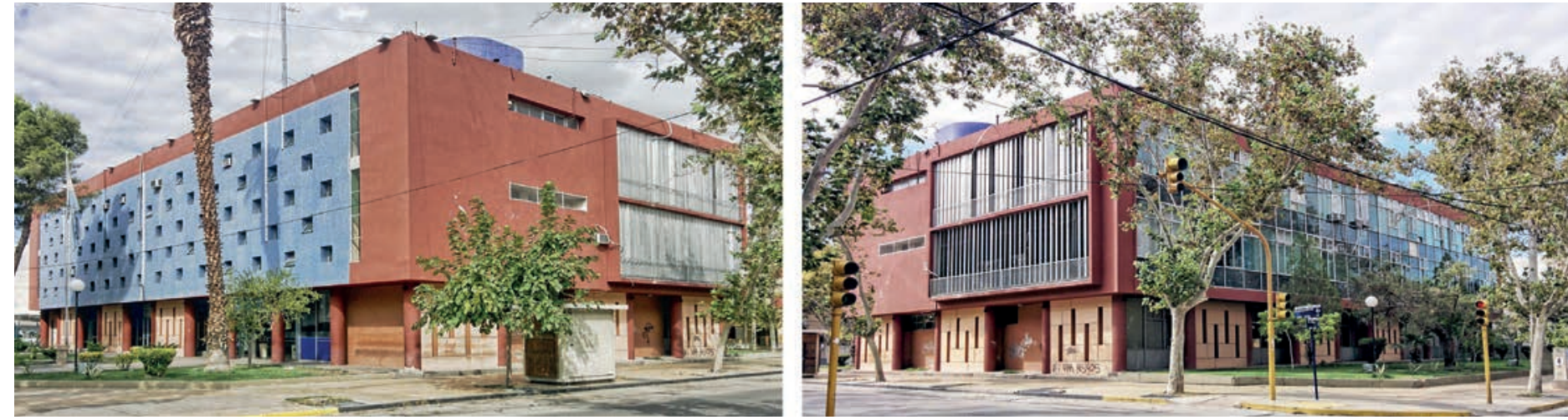

Figura 2. Izquierda: Fachada Noroeste. Derecha: Fachada Suroeste. Fuente: Elaboración de los autores.

\section{DESCRIPCIÓN DEL CASO DE ESTUDIO}

El edificio público en estudio, sede de la administración de Obras Sanitarias, Sociedad del Estado (OSSE), se ubica en la ciudad capital de San Juan, en zona bioambiental III-A de Argentina; posee clima templado cálido seco, temperatura exterior media anual de $17,2^{\circ} \mathrm{C}$ y humedad relativa media del 53\% (Instituto Argentino de Normalización y Certificación, IRAM 11603, 1996). El inmueble fue construido entre 1957 y 1962, tiene tres niveles y una superficie total de $2455 \mathrm{~m}^{2}$.

El edificio está caracterizado por la flexibilidad propia de la arquitectura del movimiento moderno e incorpora criterios de diseño bioclimático en la etapa temprana de proyecto, entre los que sobresalen: orientación del eje longitudinal en dirección Este-Oeste para un óptimo asoleamiento; parasoles móviles en fachada Este y Oeste para el control solar estacional (Figura 2); fachada Norte, con un $19 \%$ de superficie vidriada, y Sur (a modo de courting wall), con un $72 \%$, que aporta buena iluminación y ventilación natural a los espacios de trabajo. Las aberturas de la fachada Norte son mínimas y el muro, con elevada inercia térmica, permite controlar el ingreso solar del período de verano y servir de muro acumulador en invierno y verano (Figura 2).

Asimismo, el inmueble cuenta con un sistema de climatización total (HVAC) gas/eléctrico, tipo rooftop. Del relevamiento in situ, se obtiene que el equipo de refrigeración representa el 23,64\% y el de calefacción, el $7,45 \%$ de la potencia instalada total. Los datos de la demanda y el consumo específicos están descriptos en Alamino et al. (2015). De lo anterior, se advierte que un total del $44 \%$ del consumo de energía final del edificio es empleado en restituir la situación de confort del habitante. Cabe indicar que la demanda de energía eléctrica promedio anual del edificio OSSE es de 171

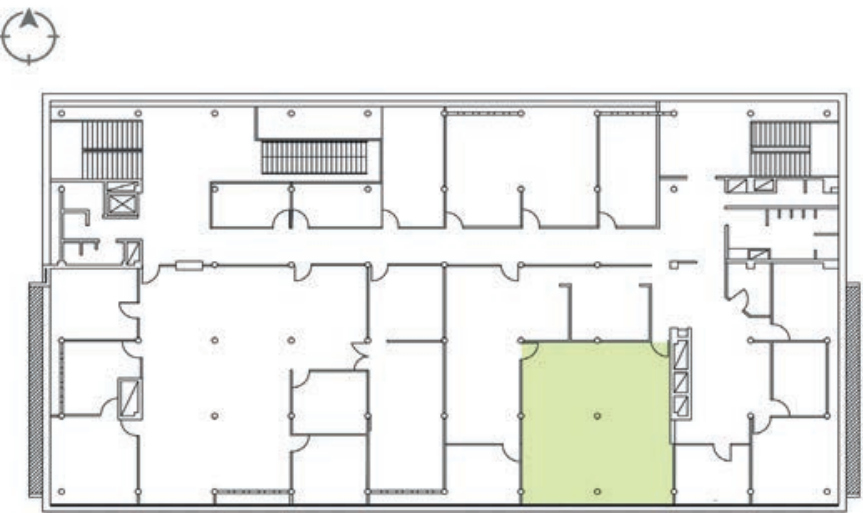

Figura 3. Planta Primer Piso de Edificio OSSE. Oficina seleccionada con orientación Sur. Fuente: Elaboración de los autores.

$\mathrm{KWh} / \mathrm{m}^{2}$.a (ibídem) y el factor de ocupación es de $3 \mathrm{~m}^{2}$ / persona.

En base a los datos de la medición in situ (ver punto 5.1) se determina una oficina promedio o tipo del edificio OSSE $\left(72 \mathrm{~m}^{2}\right)$ ubicada en el primer piso, con orientación hacia el sur, y se modela en Energy Plus con el propósito de simular y verificar los resultados de optimización para un día típico de verano (Figura 3). Energy Plus es un software de simulación para realizar análisis energéticos y térmicos en edificios. Permite calcular la cantidad de energía requerida para mantener cada espacio modelado o zona térmica a una temperatura específica. Para cada zona térmica se requiere cargar los datos climáticos del lugar, junto con la definición de los parámetros constructivos de la envolvente (muros, puertas y ventanas). También pueden detallarse el equipamiento y aparatos de consumo eléctrico junto con sus rutinas de uso. 
La configuración elegida para la simulación se corresponde con la de un "día de diseño". Para la simulación del día de diseño se debe suministrar un grupo de parámetros, tales como temperatura máxima y mínima de bulbo seco exterior, velocidad y dirección del viento, etc. En este estudio, la descripción del edificio y sus cargas internas se mantiene lo más simple posible en aras de evitar una modelización excesiva (Neto y Fiorelli, 2008).

\section{METODOLOGÍA}

\section{COMBINACIÓN ENTRE MONITOREO IN SITU(ESTUDIO DE CAMPO) Y OPTIMIZACIÓN ADAPTATIVA.}

En relación a la metodología aplicada, se propone una aproximación experimental mediante un trabajo de campo y una aproximación simulada mediante la optimización. para obtener soluciones óptimas al problema entre la eficiencia energética y el confort térmico.

Una aproximación experimental a través del trabajo de campo permite la observación in situ del edificio y su identificación como "referente" (Kuchen, 2008). El seguimiento del edificio a lo largo de su vida útil, sobre todo en el período de post-ocupación, permite corregir y optimizar el curso de su funcionamiento (EnBop, 2008). Se realiza, de esta forma, un trabajo de campo que incluye mediciones ambientales internas y externas y, en simultáneo, encuestas cortas al usuario en su espacio de trabajo. El relevamiento es de tipo transversal en verano, invierno y período transitorio y se desarrolla en diferentes oficinas elegidas aleatoriamente para cada caso de estudio, distribuidas en todos los niveles y orientaciones posibles (Kuchen, 2008).

Las mediciones se efectúan en los tres períodos desde las 8:00 hasta las 12:00 h, siguiendo un recorrido continuo. El intervalo de medición es de un minuto. En cada espacio de trabajo se consideran 3 minutos para la aclimatación de los sensores, 5 minutos adicionales para la medición y 2 minutos para el traslado del instrumental al siguiente espacio a medir. El sensor móvil se ubica en el plano de trabajo, esto es, a 0,90 $\pm 0,20 \mathrm{~m}$ sobre el nivel de piso terminado. La información objetiva (temperatura interior a bulbo seco y temperatura exterior) se toma por medio de sensores acumuladores de datos tipo Hobo U12-006 (data logger). La encuesta empleada se corresponde a la elaborada en Kuchen (2008). La misma posee dos carillas y se refiere a aspectos psicológicos, fisiológicos y físicos del usuario en relación con el ambiente interior y su actividad (Alonso Frank et al., 2015; Alamino et al., 2015). En paralelo a la medición, se lleva a cabo un relevamiento ocular. El asistente de medición vuelca en una planilla técnica datos sobre el tipo y cantidad de artefactos de consumo eléctrico, las características de la fachada, ventana, parasol, orientación, dimensiones, estado de funcionamiento del sistema de calefacción o refrigeración, ventilación, cantidad de personas, etc. En el edificio OSSE se realizaron 84 encuestas (27 mujeres y 57 hombres).

A partir de la medición in situ del edificio OSSE se define e identifica una oficina representativa de los valores promedio y se procede a la evaluación de los datos de medición y a la simulación térmico-energética. De la correlación entre los datos objetivos de las mediciones y datos subjetivos de los test, se establecen indicadores sobre el confort (en base a modelos y estándares de confort térmico referenciales, ISSO 7730, ISSO 74), así como la delimitación de rangos de aceptación y satisfacción del usuario tipo (Kuchen, 2008).

Con el fin de determinar el grado de aplicabilidad y verificación de las propuestas de optimización logradas a través de esta metodología, y así cubrir las demandas energéticas y de confort térmico, se realiza una simulación utilizando el software Energy Plus. Por medio del Ente Provincial Regulador de la Energía (EPRE), se obtiene la información sobre el consumo energético anual del edificio.

\section{MODELO DE CONFORT TÉRMICO ADOPTADO}

El trabajo de campo efectuado por Kuchen en 30 edificios de oficina en Alemania (ibídem; Kuchen y Fisch, 2009; Kuchen, Plesser y Fisch, 2012) ha permitido disponer de 1100 encuestas (votos de confort) de sensación térmica. En el método de monitoreo in situ o "spot-monitoring", las encuestas y el relevamiento de los parámetros climáticos interiores se llevan a cabo en simultáneo a fin de establecer comparaciones y detectar diversas correlaciones. Los estudios se realizan en tres tipos de edificios diferentes: con climatización natural o sin climatización (designados como Tipo 1, T1); con sistemas de climatización mixtos 0 , en inglés, mix-mode (T2); y con climatización total (T3). La ecuación que establece Kuchen en su modelo de confort térmico variable determina la capacidad de variabilidad y adaptabilidad, respectivamente, a través de la incorporación de la variable de medición en tiempo real de la temperatura operativa $\left(t_{o p}\right)$ y temperatura de neutralidad $\left(t_{n}\right)$ (ver Ecuación 1).

$$
\text { Disc. }=100-93 \cdot \exp ^{\left(-0.0009 \cdot\left(-t_{n}+t_{o p}\right)^{4}-0.034 \cdot\left(-t_{n}+t_{o p}\right)^{2}\right)}
$$

El modelo establece un máximo de $7 \%$ de disconformes posibles ante una temperatura de $23,3 \pm 1,1{ }^{\circ} \mathrm{C}$. Dentro de este rango queda delimitada la temperatura de neutralidad (Kuchen, 2008). La variabilidad de esta clase de modelo se define como la capacidad de admitir la adaptación de los usuarios hacia el ambiente térmico y asegurar la reconfiguración matemática según sea necesario, en función de las mediciones en tiempo real de las condiciones físicas, externas e internas, del edificio. Las investigaciones en Kuchen (2008) y Kuchen y Fisch (2009) evidencian que "los usuarios [...], aun en espacios con condiciones térmicas constantes, son capaces de experimentar procesos de adaptación, como, por ejemplo: modificar los niveles de 
ropa, la posición de un termostato, controlar la apertura de puertas y ventanas, ajustar un parasol, etc., pudiendo aceptar condiciones térmicas que les son impuestas" (Kuchen, Plesser y Fisch, 2012). Estas afirmaciones son referencia de otros autores dedicados al estudio de condiciones de confort en interiores (Hellwig y Bischof, 2006; Boestra, 2006; Nicol y Humphreys, 2005; de Dear, 2004 y Kuchen, Fisch y Gonzalo, 2011). Los usuarios no son receptores pasivos del ambiente térmico, sino que, por el contrario, mantienen una actitud crítica que se traduce en la habilidad de adaptación siempre posible (Kuchen, 2013, Van Hoof, 2008; Van Hoof, Mazej y Hensen, 2010).

La normativa adaptativa desarrollada en los Países Bajos, la ISSO-74, establece métodos de evaluación del confort adaptativo en base a la historia térmica de las condiciones ambientales exteriores (ISSO 74, 2004; Boerstra, Van Hoof y Van Weele, 2015). Esta, que es solo aplicable para edificios de oficina, distingue su estudio en dos tipos de edificios, los "alpha" (alto grado de control por parte de los usuarios) y los "beta" (bajo grado de control por parte de los usuarios) (Van Hoof, Mazej y Hensen, 2010).

A partir del modelo de confort térmico de Kuchen y el estándar ISSO 74, se determinan rangos de variación de la temperatura de set point interior en función de la temperatura exterior para al caso de estudio como edificio "alpha", considerando la capacidad de adaptación térmica de sus habitantes.

\section{FUNCIONES DE OPTIMIZACIÓN}

\section{Modelo de confort térmico}

La definición de la función objetivo se basa en el modelo de confort térmico de Kuchen (Ecuación 1). Determina el porcentaje de disconformes con el ambiente térmico interior en función a valores específicos de y . Definida en ASHRAE Standard 55, la temperatura operativa es una temperatura integrada que afecta globalmente la percepción térmica del usuario respecto del ambiente térmico. Según ISO 7730, la temperatura operativa combina la acción de la temperatura del aire (), la radiante media () y la velocidad del aire () sobre la percepción térmica de los usuarios.

Si $V_{a}$ se mantiene menor a $0,2 \mathrm{~m} / \mathrm{s}$ o la diferencia de temperatura entre $t_{\mathrm{a}}$ y $t_{\mathrm{rm}}$ es inferior a $4^{\circ} \mathrm{C}$, se puede aplicar la siguiente expresión:

(2) $t_{\mathrm{op}}=\left(t_{\mathrm{a}}+t_{\mathrm{rm}}\right) / 2$

Fuera de estas situaciones particulares y para el desarrollo de un cálculo más preciso de $t_{\text {op }}$, UNE-EN ISO 7730:2006 propone la siguiente ecuación:

(3) $t_{o p}=A \cdot t_{a}+(1-A) \cdot t_{r m}$
El valor del coeficiente se calcula a partir de la velocidad relativa del aire (afectada por el movimiento del cuerpo ver tabla 1) (ver ISO 7730):

si $M>1$ met,

$V_{a r}=v_{a}+0.3(M-1)$

si $M<1$ met,

$V_{\mathrm{ar}}=v_{\mathrm{a}}+0$

el coeficiente $A$ varía en función de $V_{a r}$ :

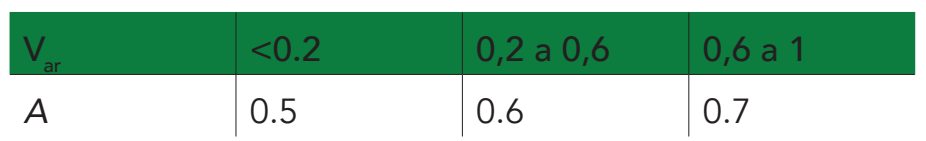

El concepto de neutralidad térmica sirve para expresar el estado físico, psicológico y fisiológico en el que el usuario percibe el ambiente térmico agradable: no siente frío, ni calor y emite un voto $\mathrm{CV}=0$ (cero) sobre la escala de 7-puntos de ASHRAE 55 (2004). De Kuchen, 2008 se obtiene la siguiente abstracción en base al modelo,

(4) $C V=b \cdot t_{o p}-a$

El criterio de neutralidad en el modelo de confort nace de considerar el enunciado de Griffiths, donde se indica que cuando $C V=0$, entonces $t_{o p}=t_{n}$ (Griffiths, 1990; Kuchen, 2008) y, por ello, es posible escribir la Ecuación 3 como sigue:

(5) $0=b \cdot t_{n}-a$

El valor de pendiente " $b$ " de la recta adquiere especial importancia ya que indica la capacidad de adaptación térmica de los habitantes para un ambiente térmico determinado. De las Ecuaciones 4 y 5, se deduce una temperatura de neutralidad, como se muestra en la Ecuación 6, donde "c" es un valor constante y puede variar en función de aspectos locales, tales como el grado del vínculo que posee el usuario entre interior y exterior, es decir, en función de las variantes de climatización y las estaciones del año (apertura de ventanas).

(6) $t_{n}=t_{o p}-(c . C V)$

donde $c=1 / b$

Asumiendo una correlación directa entre (set point de temperatura interior) y (temperatura del aire interior a bulbo seco) para el ambiente interior, esta se puede escribir de la siguiente forma, teniendo en cuenta las Ecuaciones 4 y 6 :

(7) $f_{1(\text { sp })}=$ Disc. $=100-93 \cdot \exp ^{\left(-0.0009 \cdot\left(-t_{n}+t_{o p}\right)^{4}-0.034 \cdot\left(-t_{n}+t_{o p}\right)^{2}\right)}$ 
donde $\left.-t_{n}+t_{o p}=(C V / b)=\left(s p+t_{r m}\right) / 2\right)-(a / b)$

El estándar ISSO-74 descripto anteriormente establece la siguiente ecuación para determinar en función de la (temperatura exterior), para el caso de un edificio de tipo Alpha:

(8) $t_{n}($ Alpha $)=0,31+t_{e, r e f}+17,2 \pm 2$

donde $_{\mathrm{n}}($ Alpha $)=\mathrm{Sp}$

Igualando a es posible determinar los rangos de temperatura dentro de los cuales varía el set point en función de la variación de (temperatura exterior de referencia del ambiente a bulbo seco en función a la historia térmica) para un nivel de aceptación del 90\%.

\section{Modelo de energía}

En el caso de edificios con climatización por HVAC (climatización total o mixta), las cargas por refrigeración o calefacción se producen principalmente por el balance térmico a través de la envolvente del edificio, ventilación, infiltración del aire y ganancias internas de calor. La carga neta por refrigeración se define como:

(9) $\mathrm{Q}_{\mathrm{R}}=\mathrm{Q}_{\text {env }}+\mathrm{Q}_{\text {aire, },}+\mathrm{Q}_{\text {aire, }}+\mathrm{Q}_{\mathrm{i}} \quad$ [W]

donde,

$\mathrm{Q}_{\text {env }}=$ calor sensible a través de la envolvente

$\mathrm{Q}_{\text {airess }}=$ ganancia de calor sensible por ventilación o infiltración de aire

$\mathrm{Q}_{\text {aire, }}=$ ganancia de calor latente por ventilación o infiltración de aire

$\mathrm{Q}_{\mathrm{i}}=$ ganancias internas de calor

El calor sensible a través de la envolvente se calcula a través de la siguiente expresión:

$\mathrm{Q}_{\text {env }}=\mathrm{a} / \mathrm{R}\left(\mathrm{t}_{\mathrm{e}}-\mathrm{t}_{\mathrm{a}}\right) \quad[\mathrm{W}]$

donde

$a=$ superficie del edificio

$\mathrm{R}=$ promedio de la resistencia térmica

$t=$ temperatura del aire exterior

$\mathrm{t}_{\mathrm{a}}=$ temperatura del aire interior $=\mathrm{s}_{\mathrm{p}}$

La ganancia de calor sensible por ventilación o infiltración de aire se obtiene así:

$\mathrm{Q}_{\text {aire,s }}=V \cdot p \cdot c p\left(t_{\mathrm{e}}-\mathrm{t}_{\mathrm{a}}\right) \quad[\mathrm{W}]$

donde

$\mathrm{V}=$ tasa de ventilación

$\mathrm{p}=$ densidad del aire

$\mathrm{cp}=$ calor especifico del aire
La ganancia de calor latente por ventilación o infiltración de aire (combinado con proceso interno del sistema de refrigeración) se obtiene con la siguiente fórmula:

$\mathrm{Q}_{\text {aire, }}=\mathrm{V} \cdot \mathrm{p} \cdot \mathrm{hfg}\left(\mathrm{w}_{\mathrm{e}}-\mathrm{w}_{\mathrm{i}}\right) \quad[\mathrm{W}]$

donde

$V=$ tasa de ventilación

hfg=entalpía por evaporación

$\mathrm{w}_{\mathrm{e}}=$ humedad absoluta del aire exterior

$\mathrm{w}_{\mathrm{i}}=$ humedad absoluta del aire interior

La energía consumida por el equipo por refrigeración, $C E_{R^{\prime}}$ se calcula al relacionar la carga neta por climatización con el nivel de eficiencia del sistema

(10) $f_{2}=\mathrm{CE}_{\mathrm{R}}=\mathrm{Q}_{\mathrm{R}} / \mathrm{E} f f \mathrm{c}=[\mathrm{W}]$

\section{RESULTADOS Y EVALUACIÓN}

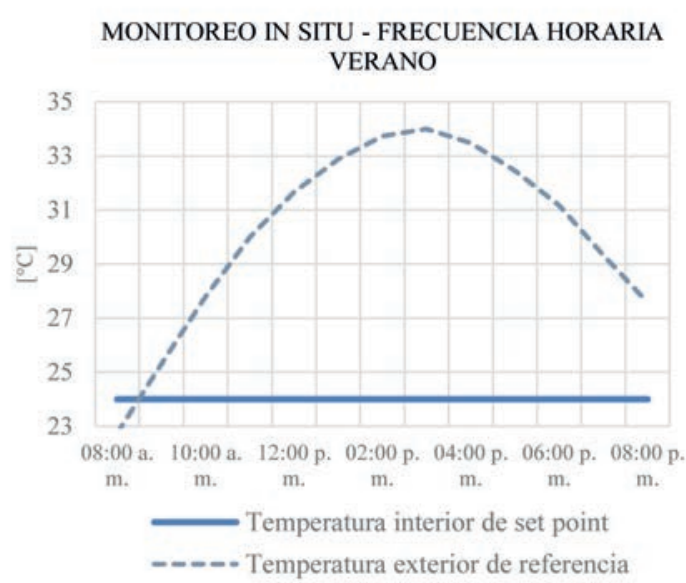

Figura 4. Resultados del monitoreo in situ en un día típico de verano con una temperatura de set point constante. Fuente: Elaboración de los autores.

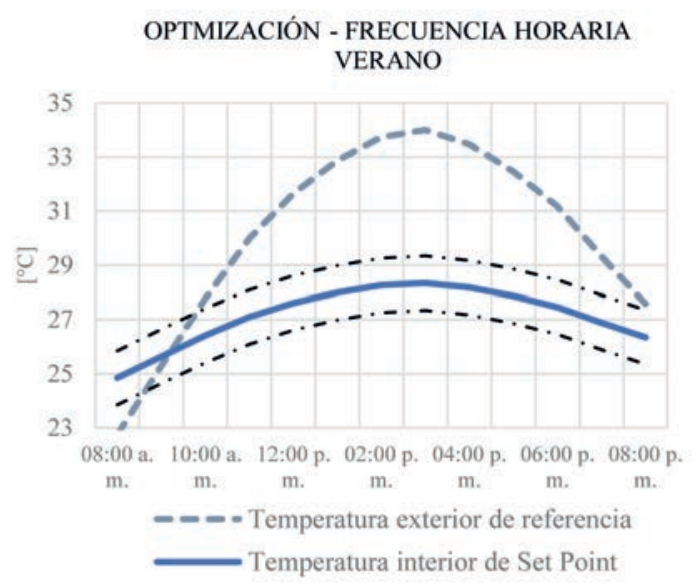

Figura 5. Variación de la temperatura de set point con optimización. Fuente: Elaboración de los autores. 
La Figura 4 presenta los resultados reales tomados de la oficina tipo para un periodo laboral definido entre las 8:00 a.m. y las 8:00 p.m. Se identifica un alto salto térmico interior exterior-interior, lo cual representa potencial de ahorro energético y justifica la optimización. Funcionando con un set point constante de $24^{\circ} \mathrm{C}$ (Figura 4), el edificio presenta un consumo de energía tipo 1 que varía de 14.5 a $20.5 \mathrm{MJ}$ (4 - 5.5 kWh / horas de trabajo) (Figura 6).

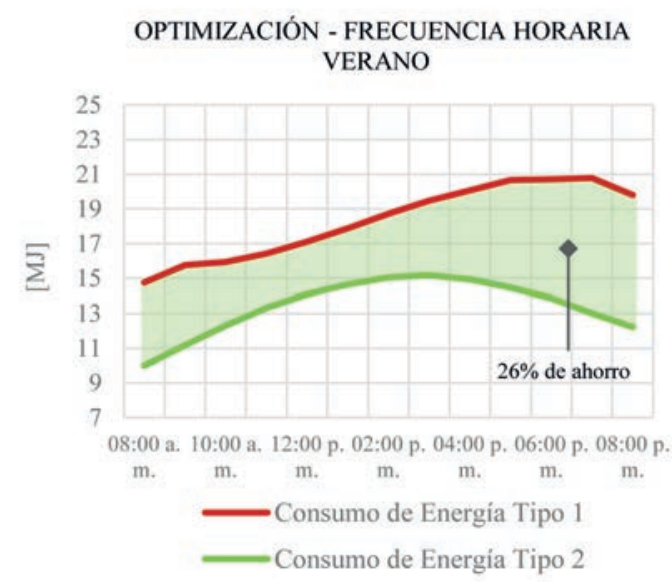

Figura 6. Optimización del consumo energético de climatización en día típico de verano. Fuente: Elaboración de los autores.

La incorporación del estándar ISSO-74 para edificios "alpha" clase A establece los rangos de posible variación del set point para mantener el ambiente interior dentro de la zona de confort. Los resultados de la simulación muestran que a través de la optimización adaptativa-variable de set point se reduce el salto térmico, manteniendo $90 \%$ de aceptabilidad (Figura 5). Se advierte, igualmente, que los habitantes permanecen en una situación de confort en un rango de temperatura que varía de $24.8^{\circ} \mathrm{C}$ a $28.3^{\circ} \mathrm{C}$, para una temperatura exterior de referencia de $22.7^{\circ} \mathrm{C}$ a $34^{\circ} \mathrm{C}$ (Figura 5). La Figura 6 evidencia que la debida consideración a la capacidad de adaptación de los habitantes tiene un impacto significativo en el consumo de energía, lo que conlleva a ahorros de energía del orden del $26 \%$ (consumo energía tipo 2) de energía eléctrica por climatización, para el período de verano.

\section{CONCLUSIONES}

El modelo adaptativo-variable aquí expuesto presenta una respuesta apropiada hacia la evaluación del confort térmico en espacios interiores de oficina, ya que considera activos a los habitantes con respecto a su ambiente térmico. La hipótesis que establece que los habitantes deben tener libertad para desarrollar sus capacidades de adaptación y tener suficiente control personal dentro de su ambiente de trabajo, se valida.
Para la optimización del confort térmico y la eficiencia energética es necesario que el modelo admita la variabilidad en el tiempo, teniendo en cuenta la evolución de las condiciones ambientales interiores y exteriores que afectan al edificio. El ahorro de energía se basa en la abstracción de que el punto de ajuste de la temperatura interior es variable y coincide con la temperatura de neutralidad dada por el modelo adaptativo de confort. En suma, la aplicación del método propuesto que combina el monitoreo in situ y la optimización adaptativa se verifica para mejorar la operación de los edificios de oficinas. Los ahorros de energía para el aire acondicionado son significativos para el período de verano, según las condiciones del presente estudio de caso.

Los estudios futuros se centrarán en el estudio de la evolución térmica horaria, aumentando la cantidad de variables analizadas para incorporarlas a las funciones de optimización de objetivos múltiples mediante algoritmos genéticos.

\section{AGRADECIMIENTOS}

Se agradece al MINCyT (Ministerio de Ciencia y la Tecnología de la Nación Argentina), al CONICET (Concejo Nacional de Investigaciones Científicas y Tecnológicas de Argentina) y a la FAUD-UNSJ (Facultad de Arquitectura, Urbanismo y Diseño - Universidad Nacional de San Juan), por el fomento a investigadores vinculados a este trabajo.

\section{REFERENCIAS BIBLIOGRÁFICAS}

AL-AJMI, Farraj F. Thermal comfort in air-conditioned mosques in the dry desert climate. Building and Environment, 2010, vol. 45, $n^{\circ} 11$, pp. 2407-2413.

ALAMINO NARANJO, Yesica; KUCHEN, Ernesto; GIL ROSTOL, Celeste, ALONSO FRANK, Alción. Monitoreo de funcionamiento y estrategias de eficiencia energética para el edificio público de Obras Sanitarias Sociedad del Estado, San Juan, Argentina. Hábitat Sustentable, 2015, vol. 5, n¹, pp. 14-23.

ALONSO FRANK, Alción, KUCHEN, Ernesto, ARBALLO, Bruno, ALAMINO NARANJO, Yesica. Influencia de la calidad ambiental edilicia y térmica del usuario en la eficiencia energética de edificios públicos. Caso de estudio: Edificio de Obras Sanitarias Sociedad del Estado, San juan - Argentina. Avances En Energías Renovables y Medio Ambiente, 2015.

ASHRAE 55:2004. Thermal environmental conditions for human occupancy (Supersedes ANSI/ASHRAE Standard 55:1992). ASHRAE Inc., Atlanta, USA, 2004.

BLIUC, Irina; ROTBERG, Rodica y DUMITRESCU, Laura. Assessing thermal comfort of dwellings in summer using EnergyPlus [en línea]. En: Proc. of the CLIMA 2007 World Congress Well Being Indoors, 2007. [Consultado 12 abril 2019]. Disponible en: http:// www.irbnet.de/daten/iconda/CIB7648.pdf 
BOESTRA, Atze. C. The adaptive thermal comfort criterion in the new EPBD IEQ Standard. BBA Indoor Environmental Consultancy, 2006.

BOERSTRA, Atze C.; VAN HOOF, Joost; VAN WEELE, A. M. A new hybrid thermal comfort guideline for the Netherlands: background and development. Architectural Science Review, 2015, vol. 58, n 1, pp. 24-34.

CHANDEL, S. S.; SHARMA, Vandna y MARWAH, Bhanu M. Review of energy efficient features in vernacular architecture for improving indoor thermal comfort conditions. Renewable and Sustainable Energy Reviews, 2016, vol. 65, pp. 459-477.

DE DEAR, R. Thermal comfort in practice. Division of Environmental and Life Sciences, Macquarie University, Australia. Indoor Air, 2004, vol. 14, n 7, pp. 32-39.

DERBEZ, Mickaël, BERTHINEAU, B., COCHET, V., PIGNON, C., RIBÉRON, J., WYART, G.... y KIRCHNER, S. A 3-year follow-up of indoor air quality and comfort in two energy-efficient houses. Building and Environment. 2014, vol. 82, pp. 288-299.

DOUNIS, Anastasios y CARAISCOS, Christos. Advanced control systems engineering for energy and comfort management in a building environment. A review. Renewable and Sustainable Energy Reviews, 2009, vol. 13, n 6-7, pp. 1246-1261.

ENBOP. Energie Betriebsoptimierung [en línea]. 2008. [Consultado 12 abril 2019]. Disponible en: http://www.enob.info.

ENVIRONMENTAL PROTECTION AGENCY [en línea]. 2014. [Consultado 12 abril 2019]. Disponible en: www.epa.gov

GRIFFITHS, I. Thermal comfort studies in buildings with passive solar features, field studies. Report to the Commission of the European Community, 1990, vol. 35.

HELLWIG, Runa Tabea y BISCHOF, Wolfgang. Gültigkeit thermischer Behaglichkeitsmodelle. Bauphysik, 2006, vol. 28, $\mathrm{n}^{\circ}$ 2, pp. 131-136.

HOLMES, Michael J. y HACKER, Jacob N. Climate change, thermal comfort and energy: Meeting the design challenges of the 21st century. Energy and Buildings, 2007, vol. 39, n 7, pp. 802-814.

INSTITUTO ARGENTINO DE NORMALIZACIÓN Y CERTIFICACIÓN (IRAM). Acondicionamiento Térmico de Edificios. Clasificación Bioambiental de la República Argentina. IRAM 11603:1996. Buenos Aires: Instituto Argentino de Normalización, ICS 91.120.10., CNA 5640, 1996.

ISO 7730. Moderate thermal environments - determination of the PMV and PPD indices and specifications of the conditions for thermal comfort, 1994.

ISSO 74. Thermische Behaaglijkeid. Publication 74, ISSO. Rotterdam, The Netherlands, 2004.

KUCHEN, Ernesto. Spot-Monitoring zum thermischen Komfort in Bürogebäuden. PHD Thesis. ISBN: 978-3-89959-783-7. Der Andere Verlag, S. 203. Tönning, Deutschland, 2008.
KUCHEN, Ernesto. Ventilación de espacios de trabajo en edificios de oficina y su influencia sobre la eficiencia energética. Hábitat Sustentable, 2013, vol. 3, n², pp. 55-65.

KUCHEN, Ernesto y FISCH, M. Norbert. Spot monitoring: thermal comfort evaluation in 25 office buildings in winter. Building and Environment, 2009, vol. 44, n 4 , pp. 839-847.

KUCHEN, Ernesto; FISCH, M. Norbert y GONZALO, Guillermo E. Modelo de Confort. Rangos de Aceptación Térmica. Avances en Energías Renovables y Medio Ambiente, 2011, vol. 14, pp. 89-96.

KUCHEN, Ernesto; PLESSER, Stefan y FISCH, M. Norbert. Eficiencia energética y confort en edificios de oficina. El caso alemán. Hábitat Sustentable, 2012, vol. 2, n², pp. 34-44.

MOROŞAN, Petru-Daniel; BOURDAIS, Romain; DUMUR, Didier y BUISSON, Jean. Building temperature regulation using a distributed model predictive control. Energy and Buildings, 2010, vol. 42, nº 9, pp. 1445-1452.

NETO, Alberto y FIORELLI, Flávio Augusto. Comparison between detailed model simulation and artificial neural network for forecasting building energy consumption. Energy and buildings, 2008, vol. 40, n²12, pp. 2169-2176.

NGUYEN, Anh-Tuan; REITER, Sigrid y RIGO, Philippe. A review on simulation-based optimization methods applied to building performance analysis. Applied Energy, 2014, vol. 113, pp. 10431058.

NICOL, J. Fergus y HUMPHREYS, Michael A. Maximum temperatures in buildings to avoid heat discomfort. En: International Conference "Passive and Low Energy Cooling for the Built Environment", Santorini, Greece, 2005.

PABLO-ROMERO, María del P.; POZO-BARAJAS, Rafael y YÑÍGUEZ, Rocío. Global changes in residential energy consumption. Energy Policy, 2017, vol. 101, pp. 342-352.

PÉREZ-LOMBARD, Luis; ORTIZ, José; POUT, Christine. A review on buildings energy consumption information. Energy and buildings, 2008, vol. 40, n³, pp. 394-398.

PROYECTO EECOM -PDTS- Res.1277/16. Eficiencia Energética y Confort en edificios públicos mediante Optimización Multiobjetivo. Director: Dr. Ernesto Kuchen. San Juan, Argentina, 2016.

SÁNCHEZ-GARCÍA, Daniel, RUBIO-BELLIDO, Carlos; MARREROMELÉNDEZ, Madelyn; GUEVARA-GARCÍA, Francisco y Canivell, Jacinto. El control adaptativo en instalaciones existentes y su potencial en el contexto del cambio climático. Hábitat Sustentable, 2017, vol.7, $\mathrm{n}^{\circ}$ 2, pp. 06-17.

STAPLES. Encuesta telefónica realizada aleatoriamente a 1000 empleados de empresas PyMEs del área de Capital y Gran Buenos Aires. Argentina, 2012.

TWEED, Christopher; DIXON, Dylan; HINTON, Emma y BICKERSTAFF, Karen. Thermal comfort practices in the home and their impact on energy consumption. Architectural Engineering and Design Management, 2014, vol. 10, n 1-2, pp. 1-24. 
VAN HOOF, Joost. Forty years of Fanger's model of thermal comfort: Comfort for all? Indoor Air, 2008, vol. 18, n³, pp. 182201.

VAN HOOF, Joost; MAZEJ, Mitja y HENSEN, Jan LM. Thermal comfort: research and practice. Frontiers in Bioscience, 2010, vol. $15, n^{\circ} 2$, pp. $765-788$.

YANG, Rui, y WANG, Lingfeng. Development of multi-agent system for building energy and comfort management based on occupant behaviors. Energy and Buildings. 2013, vol. 56, pp. 1-7. 